\title{
Developments in Environmental Transmission Electron Microscopy for Catalysis Research
}

\author{
Eric A. Stach, ${ }_{5}^{1}$ Dmitri Zakharov ${ }^{1}$, M. Cem Akatay, ${ }^{2}$ Philipp Baumann, ${ }^{3}$ Fabio Ribeiro, ${ }^{4}$ Yury Zvenich ${ }^{4}$, \\ Yuanyuan $\mathrm{Li}^{5}$, and Anatoly Frenkel ${ }^{5}$ \\ 1 Center for Functional Nanomaterials, Brookhaven National Laboratory, Upton, NY 11733 \\ 2 School of Materials Engineering and Birck Nanotechnology Center, Purdue University, West \\ Lafayette, Indiana 47907, USA \\ 3 University of Applied Sciences of Northeastern Switzerland, 4132 Muttenz, Switzerland \\ 4 Forney Hall of Chemical Engineering, Purdue University, West Lafayette, Indiana, USA \\ 5 Department of Physics, Yeshiva University, New York, NY 10016
}

Environmental transmission electron microscopy is an established technique for interrogating the interactions between solid materials and gaseous environments [1]. Two primary commercially available approaches are available, the use of differential pumping apertures to confine gases local to the sample but within the entirety of the objective lens region, and closed-cell systems which confine gases tightly around the sample region through the use of microfabricated membranes [2]. Differentially pumped systems have the advantage that the full capabilities of the instrument may be exploited during experimentation, while closed cell systems allow even higher pressures, mitigating the so-called pressure gap.

In this presentation, I will describe several instrumental advances that improve both of these approaches. In the case of differentially pumped ETEMs, it is difficult to explore specific areas of a sample during changes in reaction condition (e.g. from reductive to oxidative), because the large changes in gas composition and difficulties in smoothly altering gas flows lead to substantial sample drift, even during room temperature experimentation. We will describe our recent development of a fully integrated gas reaction system into an aberration-corrected Titan E-TEM. This system allows precise delivery of a fixed pressure gas stream to the sample, through active feedback and control. A simple Labview interface allows switching of reactive streams (with integrated safety protocols to prevent inappropriate gas mixes) and minimizes sample drift even during large gas excursions (Figure 1). This system is compatible with multiple types of sample heating systems (bulk furnace and microfabricated heating elements), and allows interrogation of the exact same sample regions during multiple reactive excursions.

This same system has been made compatible with a closed-cell TEM sample holder, and exploited for combined photon and electron experimentation. We will describe our recent studies of the conversion of ethylene by $\mathrm{Pt} / \mathrm{SiO} 2$ catalysts, based on a newly developed in-operando approach. By integrating a Clausen cell and a residual gas analyzer into the exhaust of the holder, we can directly measure the reactions occurring in the system, and confirm that the catalysts are fully functional. This system is shown to be compatible with both standard electron microscopy characterization approaches (TEM, STEM and EELS), as well as x-ray absorption spectroscopy methods (XANES, EXAFS), allowing direct links to be made between local structure and global average structure in working environments.

Finally, early observations of high temporal imaging of changes in catalyst morphology through the use of a newly installed direct detection system will be described. This system achieves frame rates of 1600 frames per second (at 512 x 512 image size), thereby greatly increasing the range of temporal resolution available in conventional in-situ experimentation. 
[1] P.L. Gai, R. Sharma and F.M. Ross, MRS Bulletin, 33, 107, 2008.

[2] J.F. Creemer, et al., Ultramic. 108, 2008.

[3] Research carried out at the Center for Functional Nanomaterials, Brookhaven National Laboratory, which is supported by the U.S. Department of Energy, Office of Basic Energy Sciences, under Contract No. DE-AC02-98CH10886. Y.L and A.F.F acknowledge additional support through the Synchrotron Catalysis Consortium, U.S. Department of Energy, Office of Basic Energy Sciences, under Contract No. DE-FG02-03ER15476. M.C.A, F.R. and Y.Z acknowledge support from the Institute for Atom-efficient Chemical Transformations (IACT), an Energy Frontier Research Center funded by the U.S. Department of Energy, Office of Science, Office of Basic Energy Sciences.

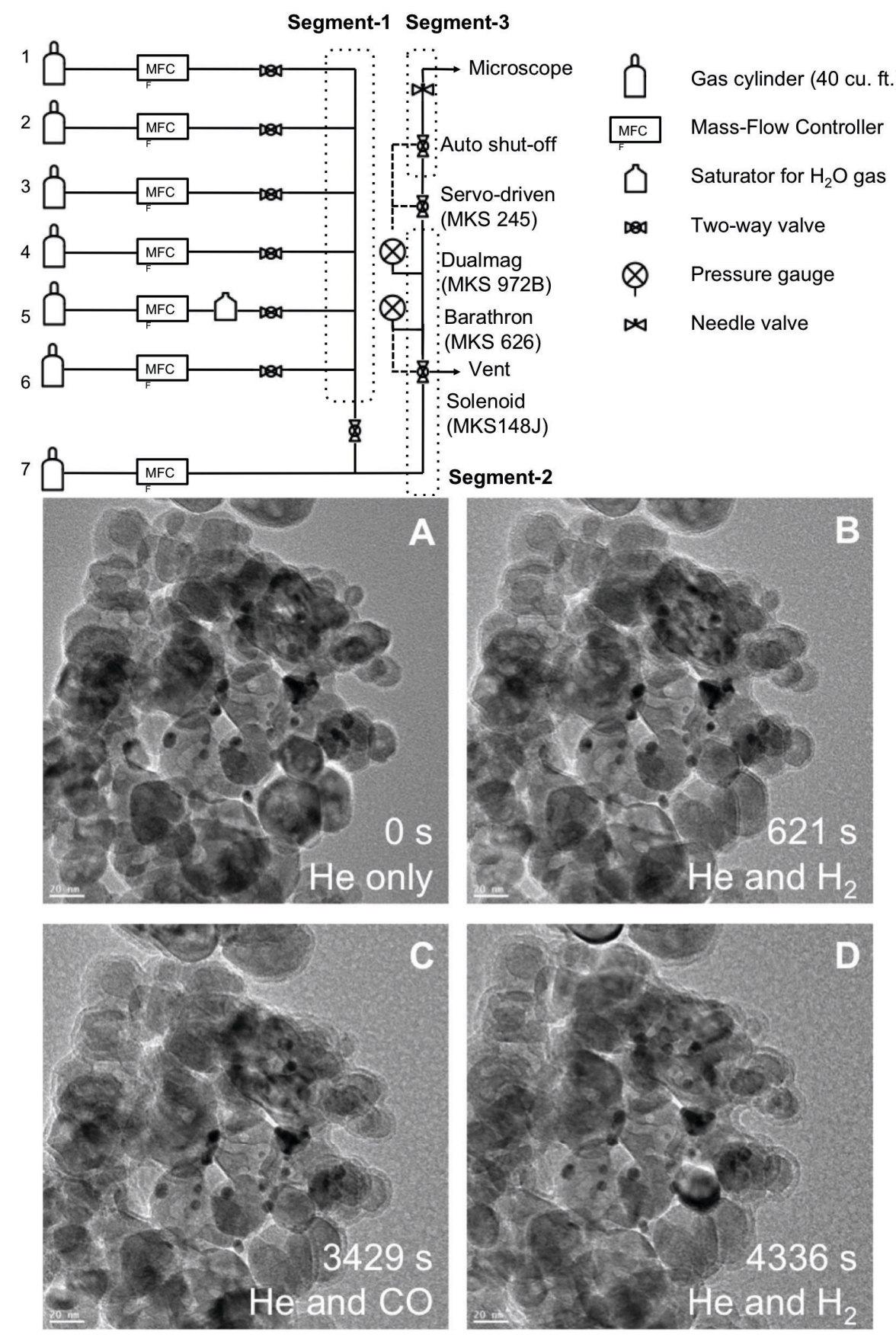

Figure 1: (above) Schematic of the gas reaction system. (A-d) TEM micrographs captured after various compositional perturbations without externally imposed changes to the sample position 